\title{
An Analogue of Covering Space Theory for Ranked Posets
}

\author{
Michael E. Hoffman \\ Dept. of Mathematics \\ U. S. Naval Academy, Annapolis, MD 21402 \\ meh@usna.edu \\ Submitted: May 10, 2001. Accepted: October 11, 2001. \\ MR Classifications: Primary 06A07,05A15; Secondary 57M10
}

\begin{abstract}
Suppose $P$ is a partially ordered set that is locally finite, has a least element, and admits a rank function. We call $P$ a weighted-relation poset if all the covering relations of $P$ are assigned a positive integer weight. We develop a theory of covering maps for weighted-relation posets, and in particular show that any weighted-relation poset $P$ has a universal cover $\widetilde{P} \rightarrow P$, unique up to isomorphism, so that
\end{abstract}

1. $\widetilde{P} \rightarrow P$ factors through any other covering map $P^{\prime} \rightarrow P$;

2. every principal order ideal of $\widetilde{P}$ is a chain; and

3. the weight assigned to each covering relation of $\widetilde{P}$ is 1 .

If $P$ is a poset of "natural" combinatorial objects, the elements of its universal cover $\widetilde{P}$ often have a simple description as well. For example, if $P$ is the poset of partitions ordered by inclusion of their Young diagrams, then the universal cover $\widetilde{P}$ is the poset of standard Young tableaux; if $P$ is the poset of rooted trees ordered by inclusion, then $\widetilde{P}$ consists of permutations. We discuss several other examples, including the posets of necklaces, bracket arrangements, and compositions.

\section{Introduction}

For topological spaces, the notion of a covering space is familiar (see, e.g., [9]): a covering map $p: X^{\prime} \rightarrow X$ is a continuous surjection such that, for sufficiently small open sets $U \subset$ $X, p^{-1}(U)$ is a disjoint union of open sets in $X^{\prime}$ each of which $p$ maps homeomorphically onto $U$. For any space $X$ satisfying appropriate hypotheses (e.g., that $X$ is connected, locally arcwise connected and semilocally simply connected), there is a simply connected covering space $\pi: \widetilde{X} \rightarrow X$, which is universal in the sense that it "factors through" any other connected cover of $X$, i.e., if $p: X^{\prime} \rightarrow X$ is any covering map with $X^{\prime}$ conneced, 
then there is a covering map $f: \widetilde{X} \rightarrow X^{\prime}$ so that $\pi=p f$. The universal covering space of $X$ is unique up to homeomorphism over $X$.

In this paper we develop a theory of covering maps for ranked posets. More precisely, we define covering maps of "weighted-relation" posets, which are locally finite ranked posets with least element that have a positive integer weight associated with each of their covering relations. We show that every such weighted-relation poset $P$ has a universal cover $\widetilde{P} \rightarrow P$, unique up to isomorphism in an appropriate category, which factors through any other cover $P^{\prime} \rightarrow P$. The universal cover $\widetilde{P}$ is "simple" in the sense that its Hasse diagram is a tree and all its covering relations have weight 1 .

In many cases where $P$ is a poset of familiar combinatorial objects, the elements of the universal cover $\widetilde{P}$ also have a simple description. For example, the poset of monomials in commuting variables $x_{1}, \ldots, x_{k}$ has a universal cover whose elements are monomials in $k$ noncommuting variables (Example 2 in $\S 4$ below); the poset of compositions (with an appropriate choice of weights) has as its universal cover the poset of Cayley permutations in the sense of [6] (Example 6). We discuss several other examples, including the posets of necklaces, bracket arrangements, partitions, and rooted trees.

\section{Weighted-relation posets}

Our terminology for posets follows $[11]$. Let $(P, \preceq)$ be a locally finite poset with least element $\hat{0}$ and rank function $|\cdot|$. By a weight system on the relations of $P$, we mean a function $n$ that assigns a nonnegative integer $n(x, y)$ to every pair $x, y \in P$ so that

1. $n(x, y) \neq 0$ if and only if $x \preceq y$;

2. for all elements $x \prec y$ and nonnegative integers $|x| \leq k \leq|y|$,

$$
n(x, y)=\sum_{|z|=k} n(x, z) n(z, y) \text {. }
$$

(Note that the second condition implies $n(x, x)=1$ for all $x \in P$.)

We call a poset $P$ together with a weight system on its relations a weighted-relation poset. By induction on $|y|-|x|$ it is easy to prove from the definition that for any $x \prec y$ in $P$

$$
n(x, y)=\sum_{x=x_{1} \prec x_{2} \prec \cdots \prec x_{k}=y} n\left(x_{1}, x_{2}\right) n\left(x_{2}, x_{3}\right) \cdots n\left(x_{k-1}, x_{k}\right),
$$

where the sum is over all saturated chains $x=x_{1} \prec x_{2} \prec \cdots \prec x_{k}=y$ from $x$ to $y$ : thus, to define $n$ it suffices to give $n(x, y)$ when $y$ covers $x$. In particular, any ranked, locally finite poset with least element can be made a weighted-relation poset by assigning 1 to every covering relation.

The motivation for this definition comes from thinking of a covering relation $x \prec y$ of $P$ as indicating $y$ can be built from $x$ by some kind of elementary operation: $n(x, y)$ is the number of ways this can be done. Then in general $n(u, v)$ is the number of ways that $v$ can be built up from $u$ via a sequence of elementary operations. For examples see $\S 4$ below. 
Let $\mathcal{W}$ be the category whose objects are weighted-relation posets, and whose morphisms are defined as follows. A morphism of weighted-relation posets $P, P^{\prime}$ is a rankpreserving function $f: P \rightarrow P^{\prime}$ such that, for any elements $t, s$ of $P$,

$$
n(f(t), f(s)) \geq \sum_{s^{\prime} \in f^{-1}(f(s))} n\left(t, s^{\prime}\right) .
$$

In particular, any such function $f$ is order-preserving. Also, if $f$ has an inverse $f^{-1}$ that is also a morphism of weighted-relation posets, then $n(f(t), f(s))=n(t, s)$ for all $t, s \in P$.

We call a weighted-relation poset $P$ simple if $n(x, y)$ is 1 or 0 for any $x, y \in P$. The following result is evident.

Proposition 2.1. If $P$ is a weighted-relation poset, the following are equivalent:

(i) $P$ is simple;

(ii) the Hasse diagram of $P$ is a tree, and every covering relation has weight 1;

(iii) for every $x \in P, n(\hat{0}, x)=1$.

We also record the following fact, which is an immediate consequence of inequality (1).

Proposition 2.2. If $f: P \rightarrow P^{\prime}$ is a morphism of weighted-relation posets and $P^{\prime}$ is simple, then $f$ is an injective function and $P$ is simple.

\section{Covering maps}

Let $P^{\prime}$ and $P$ be weighted-relation posets. We say that a rank-preserving function $\pi$ : $P^{\prime} \rightarrow P$ is a covering map if, whenever $s, r \in P$ with $\pi\left(s^{\prime}\right)=s$,

$$
n(s, r)=\sum_{r^{\prime} \in \pi^{-1}(r)} n\left(s^{\prime}, r^{\prime}\right)
$$

Note that equation (2) implies that $\pi$ is a morphism of weighted-relation posets, and taking $s=\hat{0}$, we see that $\pi$ is also surjective.

To prove that a given rank-preserving function is a covering map, it suffices to prove equation (2) for $|r|-|s|=1$. For suppose (2) holds when $|r|-|s|=1$, and suppose inductively it holds for $|r|-|s|<n, n>1$. Let $r, s \in P$ with $|r|-|s|=n$, and let $\pi\left(s^{\prime}\right)=s$. Then

$$
n(s, r)=\sum_{|t|=|s|+1} n(s, t) n(t, r)=\sum_{|t|=|s|+1} \sum_{t^{\prime} \in \pi^{-1}(t)} \sum_{r^{\prime} \in \pi^{-1}(r)} n\left(s^{\prime}, t^{\prime}\right) n\left(t^{\prime}, r^{\prime}\right),
$$

and since the sets $\pi^{-1}(t)$, as $t$ runs through the rank- $(|s|+1)$ elements of $P$, partition the rank- $(|s|+1)$ elements of $P^{\prime}$,

$$
n(s, r)=\sum_{\left|t^{\prime}\right|=\left|s^{\prime}\right|+1} \sum_{r^{\prime} \in \pi^{-1}(r)} n\left(s^{\prime}, t^{\prime}\right) n\left(t^{\prime}, r^{\prime}\right)=\sum_{r^{\prime} \in \pi^{-1}(r)} n\left(s^{\prime}, r^{\prime}\right) .
$$


If $P$ is a fixed weighted-relation poset, there is a category $\mathcal{W} / P$ of covers of $P$ whose objects are covering maps $\pi: P^{\prime} \rightarrow P$. A morphism from $\pi_{1}: P_{1} \rightarrow P$ to $\pi_{2}: P_{2} \rightarrow P$ in $\mathcal{W} / P$ is a morphism $f: P_{1} \rightarrow P_{2}$ in $\mathcal{W}$ such that $\pi_{2} f=\pi_{1}$. In fact, all such functions $f$ are covering maps.

Theorem 3.1. Suppose $\pi_{i}: P_{i} \rightarrow P$ is a covering map for $i=1,2$, and suppose $f: P_{1} \rightarrow$ $P_{2}$ is a morphism of weighted-relation posets such that $\pi_{2} f=\pi_{1}$. Then $f$ is a covering map.

Proof. We show $f$ satisfies equation (2) above. Let $s, r \in P_{2}, s^{\prime} \in P_{1}$ with $f\left(s^{\prime}\right)=s$. Since $\pi_{2}$ is a covering map,

$$
n\left(\pi_{2}(s), \pi_{2}(r)\right)=\sum_{i=1}^{k} n\left(s, r_{i}\right)
$$

where $\pi_{2}^{-1}\left(\pi_{2}(r)\right)=\left\{r_{1}, \ldots, r_{k}\right\}$. For each $r_{i}$ in the image of $f$,

$$
\sum_{r^{\prime} \in f^{-1}\left(r_{i}\right)} n\left(s^{\prime}, r^{\prime}\right) \leq n\left(s, r_{i}\right)
$$

Now $\bigcup_{i=1}^{k} f^{-1}\left(r_{i}\right)=\pi_{1}^{-1}\left(\pi_{2}(r)\right)$, and since $\pi_{1}$ is a covering map we have

$$
\sum_{r^{\prime} \in \pi_{1}^{-1}\left(\pi_{2}(r)\right)} n\left(s^{\prime}, r^{\prime}\right)=n\left(\pi_{2}(s), \pi_{2}(r)\right)=\sum_{i=1}^{k} n\left(s, r_{i}\right) .
$$

Comparing (3) and (4), we see there is a contradiction unless each of the sets $f^{-1}\left(r_{i}\right)$ is nonempty and (3) is an equality for all $i$.

Theorem 3.2. Suppose $\pi: P^{\prime} \rightarrow P$ is a covering map and $f: Q \rightarrow P$ is a morphism of weighted-relation posets, with $Q$ simple. Then $f$ can be lifted to $P^{\prime}$, i.e., there is a morphism of weighted-relation posets $f^{\prime}: Q \rightarrow P^{\prime}$ such that $\pi f^{\prime}=f$.

Proof. We define $f^{\prime}: Q \rightarrow P^{\prime}$ by induction on rank; there is no problem getting started since $f^{\prime}$ must take $\hat{0} \in Q$ to $\hat{0} \in P^{\prime}$. Suppose $f^{\prime}$ has already been defined for rank $<n$. For a rank- $(n-1)$ element $z \in Q$ and a rank- $n$ element $x \in f(Q)$ with $x \succ f(z)$, let

$$
C(x, z)=\left\{z^{\prime} \in Q\left|z^{\prime} \succ z,\right| z^{\prime} \mid=n \text {, and } f\left(z^{\prime}\right)=x\right\} .
$$

Since the Hasse diagram of $Q$ is a tree, sets of the form $C(x, z)$ partition the rank- $n$ elements of $Q$. We shall extend $f^{\prime}$ to $C(x, z)$. For $z^{\prime} \in C(x, z)$,

$$
n(f(z), x) \geq \sum_{z^{\prime} \in C(x, z)} n\left(z, z^{\prime}\right)=\operatorname{card} C(x, z) .
$$

Let $S=\left\{y \in P^{\prime} \mid y \succ f^{\prime}(z)\right.$ and $\left.\pi(y)=x\right\}$. For any $y \in S$,

$$
n(f(z), x)=n\left(\pi f^{\prime}(z), \pi(y)\right)=\sum_{y^{\prime} \in S} n\left(f^{\prime}(z), y^{\prime}\right)
$$


and hence

$$
\text { card } C(x, z) \leq \sum_{i=1}^{k} n\left(f^{\prime}(z), y_{i}\right),
$$

where $S=\left\{y_{1}, y_{2}, \ldots, y_{k}\right\}$. Choose a partition of $C(x, z)$ into disjoint subsets $S_{1}, \ldots, S_{k}$ (some possibly empty) so that $S_{i}$ has cardinality at most $n\left(f^{\prime}(z), y_{i}\right)$ : this is possible because of inequality (5). Extend $f^{\prime}$ to $C(x, z)$ by setting $f^{\prime}\left(z^{\prime}\right)=y_{i}$ for all $z^{\prime} \in S_{i}$. Then for all $z^{\prime} \in C(x, z)$,

$$
n\left(f^{\prime}(z), f^{\prime}\left(z^{\prime}\right)\right) \geq \sum_{f^{\prime}\left(z^{\prime \prime}\right)=f^{\prime}\left(z^{\prime}\right)} n\left(z, z^{\prime \prime}\right) .
$$

Reasoning in the same way as in the paragraph following equation (2) above, we can conclude that $f^{\prime}$ is extended as a morphism of weighted-relation posets; and by construction

$$
\pi f^{\prime}\left(z^{\prime}\right)=x=f\left(z^{\prime}\right)
$$

for all $z^{\prime} \in C(x, z)$.

Theorem 3.3. If $P$ is a weighted-relation poset, there is a poset $\widetilde{P}$ and a covering map $\pi: \widetilde{P} \rightarrow P$ so that $\widetilde{P}$ is a simple weighted-relation poset. Further, the fiber $\pi^{-1}(x)$ of each $x \in P$ contains $n(\hat{0}, x)$ elements.

Proof. Again we proceed by induction on the rank. Let $P^{(n)}$ be the set of elements of $P$ of rank at most $n$. Suppose a covering $\pi: \widetilde{P}^{(n-1)} \rightarrow P^{(n-1)}$ with $\widetilde{P}^{(n-1)}$ simple has already been constructed, and let $x$ be a rank- $n$ element of $P$. Since $P$ is locally finite, the set $C(x)$ of elements covered by $x$ is finite: let $C(x)=\left\{x_{1}, \ldots, x_{r}\right\}$. Each fiber $\pi^{-1}\left(x_{i}\right)$ contains $n\left(\hat{0}, x_{i}\right)$ rank- $(n-1)$ elements of $\widetilde{P}$ : call them $\widetilde{x}_{i 1}, \widetilde{x}_{i 2}, \ldots, \widetilde{x}_{i m_{i}}$, where $m_{i}=n\left(\hat{0}, x_{i}\right)$. Let $K(x)$ be the set

$$
\left\{(i, j, k) \mid 1 \leq i \leq \operatorname{card} C(x), 1 \leq j \leq n\left(\hat{0}, x_{i}\right), 1 \leq k \leq n\left(x_{i}, x\right)\right\}
$$

and define

$$
\widetilde{P}^{(n)}=\widetilde{P}^{(n-1)} \cup \coprod_{x \in P,|x|=n} K(x) .
$$

Extend the weight system (and order) of $\widetilde{P}^{(n-1)}$ to $\widetilde{P}^{(n)}$ by putting

$$
n(z,(i, j, k))= \begin{cases}1, & \text { if } z \preceq \widetilde{x}_{i j}, \\ 0, & \text { otherwise }\end{cases}
$$

for any $(i, j, k) \in K(x), z \in \widetilde{P}^{(n-1)}$. Then $\widetilde{P}^{(n)}$ is simple: for any $(i, j, k) \in K(x)$ there is a unique chain to $\hat{0}$ passing through $\widetilde{x}_{i j}$, so

$$
n(\hat{0},(i, j, k))=n\left(\hat{0}, \widetilde{x}_{i j}\right) n\left(\widetilde{x}_{i j},(i, j, k)\right)=1 .
$$

(The set $C(x) \cap C\left(x^{\prime}\right)$ may be nonempty for $x \neq x^{\prime}$, so the same point of $\widetilde{P}^{(n-1)}$ may be labelled as both $\widetilde{x}_{i j}$ and $\widetilde{x}_{p q}^{\prime}$, but this does not affect the conclusion since we are taking a disjoint union of the $K(x)$.) 
Now extend $\pi$ to $\widetilde{P}^{(n)}$ by having $\pi$ send each element of $K(x)$ to $x$. Then $\pi^{-1}(x)=$ $K(x)$ contains

$$
\sum_{i=1}^{r} n\left(\hat{0}, x_{i}\right) n\left(x_{i}, x\right)=n(\hat{0}, x)
$$

elements. Also, for any $z \in \widetilde{P}^{(n-1)}$ and rank-n element $x$ of $P$, we have

$$
\begin{aligned}
n(\pi(z), x)=\sum_{i=1}^{r} n\left(\pi(z), x_{i}\right) n\left(x_{i}, x\right) & = \\
\sum_{i=1}^{r} \sum_{j=1}^{m_{i}} \sum_{k=1}^{n\left(x, x_{i}\right)} n\left(z, \widetilde{x}_{i j}\right) n\left(\widetilde{x}_{i j},(i, j, k)\right) & =\sum_{w \in \pi^{-1}(x)} n(z, w),
\end{aligned}
$$

so $\pi$ is extended as a covering map.

By a universal cover of $P$, we mean a cover $\widetilde{P} \rightarrow P$ so that, for any other cover $P^{\prime} \rightarrow P$, there is a morphism of $\mathcal{W} / P$ from $\widetilde{P} \rightarrow P$ to $P^{\prime} \rightarrow P$.

Theorem 3.4. If $P$ is a weighted-relation poset, a cover $\widetilde{P} \rightarrow P$ is universal if and only if $\widetilde{P}$ is simple, and such a cover is unique up to isomorphism in $\mathcal{W} / P$.

Proof. Suppose that $p: \widetilde{P} \rightarrow P$ is a cover with $\widetilde{P}$ simple, and let $\pi: P^{\prime} \rightarrow P$ be another cover. By Theorem 3.2, $p$ can be lifted to a morphism $p^{\prime}: \widetilde{P} \rightarrow P^{\prime}$ of weighted-relation posets so that $\pi p^{\prime}=p$ : but this means $p: \widetilde{P} \rightarrow P$ is a universal cover. Thus, a simple cover is universal.

Now suppose $\pi^{\prime}: P^{\prime} \rightarrow P$ is a universal cover. By Theorem 3.3 there is a simple cover $\pi: \widetilde{P} \rightarrow P$, and by universality there is a morphism of $\mathcal{W} / P$ from $\pi^{\prime}$ to $\pi$. Thus there is a morphism of weighted posets $f: P^{\prime} \rightarrow \widetilde{P}$ which (by Theorem 3.1) is a covering map, hence surjective; and since $\widetilde{P}$ is simple, Proposition 2.2 says $f$ is injective and $P^{\prime}$ is simple. It follows that $f$ is an isomorphism of $\mathcal{W} / P$.

\section{Examples}

Example 1. Let $P$ be the poset of subsets of $\{1,2, \ldots, n\}$, ordered by inclusion, with each covering relation given weight 1 . Then the universal cover $\widetilde{P}$ can be identified with the set of linearly ordered subsets of $\{1,2, \ldots, n\}$, with $A \preceq B$ in $\widetilde{P}$ if $A$ is an initial segment of $B$; and $\widetilde{P} \rightarrow P$ forgets the order. Evidently the fiber of any rank- $k$ element of $P$ has $k$ ! elements, so there are a total of $k !\left(\begin{array}{l}n \\ k\end{array}\right)$ rank- $k$ elements in $\widetilde{P}$.

Example 2. Let $M$ be the poset of monomials in $k$ commuting variables $x_{1}, \ldots, x_{k}$, with $m \preceq m^{\prime}$ in $M$ if there is a monomial $m^{\prime \prime}$ such that $m^{\prime}=m m^{\prime \prime}$. The rank on $M$ is given by total degree, each of the $x_{i}$ having degree one; the least element of $M$ is the empty monomial 1; and the covering relations are all given weight 1 . Then the universal cover 
$\widetilde{M}$ is isomorphic to the poset of monomials in $k$ noncommuting variables $X_{1}, \ldots, X_{k}$, with weights given by

$$
n\left(w, w^{\prime}\right)= \begin{cases}1, & \text { if } w^{\prime}=w X_{i} \text { for some } i, \\ 0, & \text { otherwise }\end{cases}
$$

for $\left|w^{\prime}\right|-|w|=1$. Clearly $\widetilde{M}$ is simple. The function $\pi: \widetilde{M} \rightarrow M$ that sends $X_{i}$ to $x_{i}$ (so, e.g., $\left.\pi^{-1}\left(x_{1}^{2} x_{2}\right)=\left\{X_{1}^{2} X_{2}, X_{1} X_{2} X_{1}, X_{2} X_{1}^{2}\right\}\right)$ is a covering map. The cardinality of the fiber of any monomial is given by

$$
n\left(1, x_{1}^{i_{1}} x_{2}^{i_{2}} \cdots x_{k}^{i_{k}}\right)=\left(\begin{array}{c}
i_{1}+\cdots+i_{k} \\
i_{1} i_{2} \cdots i_{k}
\end{array}\right)
$$

and the total number of rank- $n$ elements of $\widetilde{M}$ is

$$
\sum_{i_{1}+\cdots+i_{k}=n}\left(\begin{array}{ccc}
n \\
i_{1} & \cdots & i_{k}
\end{array}\right)=k^{n} .
$$

Example 3. Let $\mathcal{N}$ be the set of circular necklaces made of beads of $k$ colors: a rank- $m$ element of $\mathcal{N}$ is a necklace with $m$ beads, and the least element is the empty necklace $\emptyset$. For a rank- $(m-1)$ necklace $p$ and a rank- $m$ necklace $q, p \prec q$ if $q$ can be obtained from $p$ by insertion of a bead of any color, and $n(p, q)$ is the number of ways to insert a bead into $p$ to get $q$. For example, in the case $k=2$,

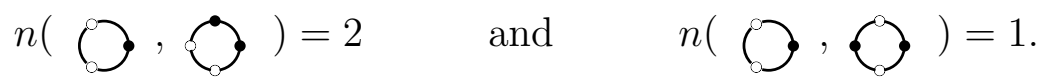

The universal cover $\tilde{\mathcal{N}}$ can be described as the poset of necklaces with labelled beads, i.e., the beads of a rank- $m$ necklace are labelled $1,2 \ldots, m$, with $\widetilde{\mathcal{N}} \rightarrow \mathcal{N}$ the function that forgets the labels. It is clear that $\tilde{\mathcal{N}}$ is simple, since there is a unique chain from any labelled necklace to $\emptyset$ via the operation of removing the highest-label bead. A rank- $m$ element of $\widetilde{\mathcal{N}}$ can be thought of as a " $k$-colored permutation" mod rotation, so there are $k^{m}(m-1)$ ! such elements. Also, the fiber of a given necklace $p \in \mathcal{N}$ with $m$ beads has $n(\emptyset, p)=m ! / N(p)$ elements, where $N(p)$ is the number of rotations that take $p$ to itself (necessarily a divisor of $m$ ): $p$ is called primitive if $N(p)=1$. Evidently a necklace $p$ with $N(p)=d$ has a primitive "quotient necklace" of size $\frac{m}{d}$. Thus, if $P(m)$ is the number of primitive necklaces of size $m$, we have

$$
\sum_{d \mid m} P\left(\frac{m}{d}\right) \frac{m !}{d}=\sum_{|p|=m} n(\emptyset, p)=k^{m}(m-1) !,
$$

or $\sum_{d \mid m} P(d) d=k^{m}$. By Möbius inversion we obtain the classical result

$$
P(m)=\frac{1}{m} \sum_{d \mid m} \mu(d) k^{\frac{m}{d}}
$$

Cf. [7, Theorem 7.1]. 
Example 4. Let $\mathcal{B}$ be the set of balanced bracket arrangements: a rank-n element of $\mathcal{B}$ is a sequence of $n$ left brackets and $n$ right brackets so that, reading left to right, the number of right brackets never exceeds the number of left brackets. For $b, b^{\prime} \in \mathcal{B}$ with $\left|b^{\prime}\right|-|b|=1$, let $n\left(b, b^{\prime}\right)$ be the number of ways to insert a balanced pair \langle\rangle into $b$ to obtain $b^{\prime}$, e.g., $n(\langle\rangle\langle\rangle,\langle\langle\rangle\rangle\langle\rangle)=1$ and $n(\langle\rangle\langle\rangle,\langle\rangle\langle\rangle\langle\rangle)=3$. The least element is the empty arrangement $\emptyset$. Then $\mathcal{B}$ is a weighted-relation poset.

The universal cover $\widetilde{\mathcal{B}}$ has rank- $n$ elements that are permutations $a_{1} a_{2} \cdots a_{2 n}$ of the multiset $\{1,1,2,2, \ldots, n, n\}$ such that, if $a_{i}>a_{j}$ and $i<j$, then there is some $k<j, k \neq i$, with $a_{k}=a_{i}$. In particular, if $s$ is a rank- $n$ element of $\widetilde{\mathcal{B}}$, then the two occurrences of $n$ in $s$ must be adjacent. We define a partial order on $\widetilde{\mathcal{B}}$ by declaring that the rank- $n$ element $a_{1} a_{2} \ldots a_{2 n}$ covers the rank- $(n-1)$ element $a_{1} \cdots a_{i-1} a_{i+2} \cdots a_{2 n}$, where $a_{i}=a_{i+1}=n$, and define the weight of all covering relations to be 1 . Then $\widetilde{\mathcal{B}}$ is evidently simple.

Define $\pi: \widetilde{\mathcal{B}} \rightarrow \mathcal{B}$ by sending $s \in \widetilde{\mathcal{B}}$ to the bracket arrangement obtained by replacing the first occurrence of each positive integer in $s$ by $\langle$, and the second occurrence of each positive integer by $\rangle$. Let $s$ be a rank- $(n-1)$ element of $\mathcal{B}$, with $\pi\left(s^{\prime}\right)=s$. Then a rank- $n$ element $r^{\prime} \succ s^{\prime}$ is obtained by inserting $n n$ into $s^{\prime}$, corresponding to inserting \langle\rangle into $s$. Thus, for any $r \succ s$ in $\mathcal{B}$ with $|r|-|s|=1$,

$$
\begin{aligned}
n(s, r) & =\text { number of ways to insert }\langle\rangle \text { into } s \text { to get } r \\
& =\sum_{r^{\prime} \in \pi^{-1}(r)} n\left(s^{\prime}, r^{\prime}\right)
\end{aligned}
$$

so $\pi$ is a covering map.

It is well known that there are $C_{n}$ rank- $n$ elements of $\mathcal{B}$, where

$$
C_{n}=\frac{1}{n+1}\left(\begin{array}{c}
2 n \\
n
\end{array}\right)
$$

is the $n$th Catalan number. The number of rank- $n$ elements of $\widetilde{\mathcal{B}}$ can be seen to be

$$
(2 n-1) ! !=(2 n-1)(2 n-3) \cdots 3 \cdot 1
$$

as follows. If $s=a_{1} a_{2} \cdots a_{2 n} \in \widetilde{\mathcal{B}}$, there are $2 n-1$ possible choices of $i$ so that $a_{i}$ is the first occurrence of $n$ in $s$. Once $i$ is chosen, then $a_{i+1}=n$, so $s$ covers the rank- $(n-1)$ element $a_{1} \cdots a_{i-1} a_{i+2} \cdots a_{2 n}$ of $\widetilde{\mathcal{B}}$, which by induction can be chosen in $(2 n-3)$ !! ways. The phenomenon that labelling elements of a set enumerated by Catalan numbers gives a set enumerated by double factorials was noted in [3].

Example 5. Let $\mathcal{F}$ be the set of partitions of nonnegative integers, ordered by inclusion of their Young diagrams. Thus, a partition $\lambda$ of $n$ covers a partition $\mu$ of $n-1$ if $\lambda$ can be obtained from $\mu$ by increasing one part of $\mu$ by 1 , or by adding a new part of size 1 to $\mu$ : and we assign weight 1 to every covering relation. Then a rank- $n$ element of the universal cover $\widetilde{\mathcal{F}}$ is a Young diagram with boxes labelled $1,2 \ldots, n$ so that the labels increase from left to right and from top to bottom, i.e., a standard Young tableau. The ordering on $\widetilde{F}$ is by inclusion, and $\widetilde{\mathcal{F}} \rightarrow \mathcal{F}$ is the obvious function. The cardinality $n(\emptyset, \lambda)$ of the fiber of 
a partition $\lambda$ is given by the hook-length formula (see [12, Cor. 7.21.6]). More generally, when $\mu \prec \lambda$ the number $n(\mu, \lambda)$ counts standard Young tableaux of skew shape $\lambda / \mu$ (see [12, Cor. 7.16.3] for a formula). There is also an algebraic interpretation of the numbers $n(\mu, \lambda)$ : if we let $s_{\lambda}$ be the Schur symmetric function corresponding to the partition $\lambda$, then

$$
s_{1}^{k} s_{\mu}=\sum_{|\lambda|=|\mu|+k} n(\mu, \lambda) s_{\lambda}
$$

(see [12, Sect. 7.15]).

Example 6. Let $\mathcal{C}$ be the poset of compositions, i.e., finite sequences of integers, with rank given by the sum, and least element $\emptyset$. For compositions $I, J$ with $|J|-|I|=1$, we define

$$
n(I, J)= \begin{cases}1, & \text { if } J \text { is obtained from } I \text { by increasing one part; } \\ m, & \text { if there are } m \text { ways to insert } 1 \text { into } I \text { to get } J \\ 0, & \text { otherwise. }\end{cases}
$$

Thus, e.g., $n(121,122)=1, n(121,1121)=2$, and $n(121,212)=0$. This defines a weight system of $\mathcal{C}$, so $\mathcal{C}$ is a weighted-relation poset.

A rank- $n$ element of the universal cover $\widetilde{\mathcal{C}}$ is a Cayley permutation of length $n$ as defined in [6], i.e., a length- $n$ sequence $s$ of positive integers such that any positive integer $i<j$ appears in $s$ whenever $j$ does. The partial order on $\widetilde{\mathcal{C}}$ is defined as follows. If $s=a_{1} \cdots a_{n}$ is a Cayley permutation, let $m(s)=\max \left\{a_{1}, \ldots, a_{n}\right\}$. Then $s$ covers $a_{1} \cdots a_{n-1}$ if the latter is a Cayley permutation: otherwise, $s$ covers $p\left(a_{1}\right) \cdots p\left(a_{n-1}\right)$, where $p$ is the orderpreserving bijection from $\left\{a_{1}, \ldots, a_{n-1}\right\}$ to $\{1,2, \ldots, m(s)-1\}$. For example, the order ideal generated by 41332 is

$$
41332 \succ 3122 \succ 312 \succ 21 \succ 1 \succ \emptyset .
$$

If we give each covering relation weight 1 , then $\widetilde{\mathcal{C}}$ is evidently simple.

Let $\pi: \widetilde{\mathcal{C}} \rightarrow \mathcal{C}$ be the function that sends a sequence $s$ to the sequence $i_{1} i_{2} \cdots i_{k}$, where $i_{j}$ is the number of times $j$ occurs in $s$; e.g., $\pi^{-1}(13)=\{1222,2122,2212,2221\}$. To see that $\pi$ is a covering map, consider compositions $I, J$ with $|J|=|I|+1$. Let $I=i_{1} \cdots i_{k}$ and $s=a_{1} \cdots a_{n} \in \widetilde{\mathrm{C}}$ with $\pi(s)=I$. Suppose first that $J$ is obtained from $I$ by increasing the size of one part, so $J=i_{1} \cdots i_{r-1}\left(i_{r}+1\right) i_{r+1} \cdots i_{k}$. Then $n(I, J)=1$ and there is only one $t \succ s$ with $\pi(t)=J$, namely $t=a_{1} \cdots a_{n} r$. Now suppose $J$ is obtained from $I$ by inserting 1 , i.e., $J=i_{1} \cdots i_{r} 1 i_{r+1} \cdots i_{k}$; without loss of generality we can assume $i_{r} \neq 1$. Then $J$ contains a string of 1 's of length $n(I, J)$ after $i_{r}$. The possible elements $t \succ s$ in $\widetilde{\mathcal{C}}$ with $\pi(t)=J$ are of the form $t=q\left(a_{1}\right) q\left(a_{2}\right) \cdots q\left(a_{n}\right)(r+i)$, where $i$ runs from 1 to $n(I, J)$ and $q$ is the order-preserving bijection from $\left\{a_{1}, \ldots, a_{n}\right\}=\{1, \ldots, k\}$ to $\{1, \ldots, r+i-1, r+i+1, \ldots, k+1\}$. Finally, if $n(I, J)=0$, we must have $n(s, t)=0$ for any $t \in \widetilde{\mathcal{C}}$ with $\pi(t)=J$ since the previous two cases have exhausted all the possibilities for $t$ to cover $s$. So in any case,

$$
n(I, J)=\sum_{t \in \pi^{-1}(J)} n(s, t)
$$


when $|J|-|I|=1$ and $\pi(s)=I$.

The cardinality of $n(\emptyset, I)$ of the fiber of a composition $I=i_{1} \cdots i_{k}$ is evidently the multinomial coefficient

$$
\left(\begin{array}{ccc} 
& |I| & \\
i_{1} & \cdots & i_{k}
\end{array}\right)
$$

There is an algebraic interpretation of the numbers $n(I, J)$ analogous to that of the preceding example: if $M_{I}$ is the monomial quasisymmetric function corresponding to the composition $I$ (see [7, Sect. 9.4], or [12, Sect. 7.19] for definitions), then

$$
M_{1}^{k} M_{I}=\sum_{|J|=|I|+k} n(I, J) M_{J} .
$$

In particular, the multinomial coefficients $n(\emptyset, J)$ appear in the expansion of $M_{1}^{k}$.

Example 7. Let $\mathcal{T}$ be the poset of rooted trees ordered by inclusion, i.e., $t^{\prime} \succ t$ if $t^{\prime}$ can be obtained from $t$ by adding new edges and vertices. The rank function is given by

$$
|t|=\text { number of vertices of } t-1 \text {, }
$$

and the least element is the tree - consisting of the root vertex. The weight system is defined as follows: if $\left|t^{\prime}\right|-|t|=1$, let $n\left(t, t^{\prime}\right)$ be the number of vertices of $t$ to which a new edge and terminal vertex may be added to obtain $t^{\prime}$.

Rank- $n$ elements of $\widetilde{\mathcal{T}}$ are permutations of $\{1,2, \ldots, n\}$. A permutation $\sigma=s_{1} s_{2} \cdots s_{n}$ of $\{1, \ldots, n\}$ with $s_{i}=n$ covers the permutation

$$
\tau=s_{1} \cdots s_{i-1} s_{i+1} \cdots s_{n}
$$

of $\{1, \ldots, n-1\}$ (and no other). The least element is the empty permutation $\emptyset$. Then $\tilde{\mathcal{T}}$ is clearly simple if we give each covering relation weight 1 .

Now we define the covering map $\pi: \widetilde{\mathcal{T}} \rightarrow \mathcal{T}$. Let $\pi(\emptyset)=\bullet$, and given a nonempty permutation $\sigma=s_{1} s_{2} \cdots s_{n}$ define a rooted tree with vertices labelled $0,1, \ldots, n$ as follows. Label the root 0 , and attach the vertex labelled $i$ to the vertex labelled $j<i$ if $j$ is the last element of the sequence $s_{1} s_{2} \ldots s_{k-1}$ that is smaller than $i$, where $s_{k}=i$; attach $i$ to the root if no such $j$ exists. This associates a labelled rooted tree with each $\sigma \in \widetilde{\mathcal{T}}$, and $\pi(\sigma)$ is just the rooted tree obtained by forgetting the labels. Thus, e.g.,

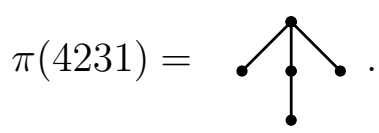

To see that $\pi$ is a covering map, note first that terminal vertices of $\pi(\sigma)$ correspond either to descents of $\sigma$ (i.e., terms $s_{i}$ with $s_{i}>s_{i+1}$ ), or to the final term. Now a permutation $\sigma$ with $|\sigma|=n$ covers $\tau$ exactly when $\sigma$ is obtained by inserting $n$ into $\tau$, e.g., $2413 \succ 213$. This always introduces a new descent (or new final term) into $\tau$, and corresponds to adding a new edge and terminal vertex to $\pi(\tau)$; moreover, the $n$ possible 
places to insert $n$ in a rank- $(n-1)$ permutation $\tau$ correspond to the $n$ vertices of $\pi(\tau)$ where a new edge and vertex can be attached. Thus, for trees $r, s$ with $|r|-|s|=1$ and $s=\pi(\tau)$,

$$
\begin{aligned}
n(s, r) & =\text { number of permutations } \sigma \succ \tau \text { with } \pi(\sigma)=r \\
& =\sum_{\sigma \in \pi^{-1}(r)} n(\tau, \sigma) .
\end{aligned}
$$

The cardinality $n(\bullet, t)$ of the fiber of a rank- $n$ rooted tree $t$ is the number of distinct labelled rooted trees (with labels coming from $\{0,1, \ldots, n\}$ and strictly increasing as one moves away from the root) that are isomorphic to $t$ when the labels are removed. Since there are $n$ ! rank- $n$ elements of $\widetilde{\mathcal{T}}$,

$$
\sum_{|t|=n} n(\bullet, t)=n ! .
$$

In fact, if we let $e(t)$ be the number of terminal vertices of the tree $t$ we have

$$
\sum_{|t|=n, e(t)=k} n(\bullet, t)=\left\langle\begin{array}{c}
n \\
k-1
\end{array}\right\rangle,
$$

where $\left\langle\begin{array}{l}p \\ q\end{array}\right\rangle$ is the number of permutations of $\{1, \ldots, p\}$ with $q$ descents (Eulerian number), because of the correspondence between descents of $\sigma$ and terminal vertices of $\pi(\sigma)$. Cf. [11, Prop. 1.3.16].

The numbers $n(\bullet, t)$ appear in connection with the "growth operator" on the Hopf algebra of rooted trees studied by Connes and Kreimer [2]. In [1, 5], $n(\bullet, t)$ is called the "Connes-Moscovici weight" of $t$, and some results about it are obtained. To describe them requires a few definitions. Given a tree $t$, let $V(t)$ be the set of vertices of $t$, and for $v \in V(t)$ let $t_{v}$ be the subtree consisting of $v$ and all its descendents (with $v$ as root): thus $t_{r}=t$ if $r$ is the root of $t$, and $t_{v}=\bullet$ if $v$ is a terminal vertex. Define the "tree factorial" of $t$ by

$$
t !=\prod_{v \in V(t)}\left(\left|t_{v}\right|+1\right)
$$

Also, if $v_{1}, \ldots, v_{k}$ are the children of the root of $t$, define the symmetry group $S G(t)$ to be the group of permutations $\sigma$ of $\{1, \ldots, k\}$ such that $t_{v_{i}}$ and $t_{v_{j}}$ are isomorphic rooted trees when $\sigma(i)=j$. Define the symmetry degree of $t$ to be

$$
S_{t}=\prod_{v \in V(t)} \operatorname{card} S G\left(t_{v}\right) .
$$

For example, the tree $t=\pi(4231)$ above has $t !=10$ and $S_{t}=2$. In [1] it is shown that

$$
n(\bullet, t)=\frac{(|t|+1) !}{t ! S_{t}} .
$$


Equation (6) is actually a variant of the generalized hook-length formula for rooted trees that appears in [10, Sect. 22], [4, Ex. 5.1.4-20], and [8]. To see this, note that if $T$ is a realization of the rooted tree $t$ as a planar directed graph (with arrows coming out from the root), then the number of ways to attach the labels $\{0,1, \ldots,|t|\}$ to the vertices of $T$ so that the labels strictly increase outward from the root is $n(\bullet, t) S_{t}$. Also of interest is the result of [5] that

$$
\sum_{|t|=n} \frac{n(\bullet, t)}{t !}=\frac{n !}{2^{n}}
$$

\section{References}

[1] D. J. Broadhurst and D. Kreimer, Renormalization automated by Hopf algebra, J. Symbolic Comput. 27 (1999), 581-600.

[2] A. Connes and D. Kreimer, Hopf algebras, renormalization, and noncommutative geometry, Comm. Math. Phys. 199 (1998), 203-242.

[3] M. R. T. Dale and J. W. Moon, The permuted analogues of three Catalan sets, J. Statis. Plann. Inference 34 (1993), 75-87.

[4] D. E. Knuth, The Art of Computer Programming, vol. 3, 2nd ed., Addison-Wesley, Reading, Mass., 1998.

[5] D. Kreimer, Chen's iterated integral represents the operator product expansion, Adv. Theor. Math. Phys. 3 (2000), 227-270.

[6] M. Mor and A. S. Fraenkel, Cayley permutations, Discrete Math. 48 (1984), 101-112.

[7] C. Reutenauer, Free Lie Algebras, Oxford University Press, New York, 1993.

[8] B. E. Sagan, Enumeration of partitions with hooklengths, European J. Combin. 3 (1982), 85-94.

[9] E. Spanier, Algebraic Topology, McGraw-Hill, New York, 1966.

[10] R. P. Stanley, Ordered Structures and Partitions, Memoirs Amer. Math. Soc. No. 119, American Mathematical Society, Providence, R.I., 1972.

[11] R. P. Stanley, Enumerative Combinatorics, vol. 1, Wadsworth and Brooks/Cole, Monterey, California, 1986.

[12] R. P. Stanley, Enumerative Combinatorics, vol. 2, Cambridge University Press, New York, 1999. 